IZA DP No. 2324

\title{
Identification of Peer Effects
}

Using Group Size Variation

\author{
Laurent Davezies \\ Xavier d'Haultfoeuille \\ Denis Fougère
}

September 2006 


\title{
Identification of Peer Effects Using Group Size Variation
}

\author{
Laurent Davezies \\ DEPP and CREST-INSEE \\ Xavier d'Haultfoeuille \\ ENSAE, CREST-INSEE \\ and Université Paris I-Panthéon-Sorbonne \\ Denis Fougère \\ CNRS, CREST-INSEE, CEPR \\ and IZA Bonn
}
Discussion Paper No. 2324
September 2006

\author{
IZA \\ P.O. Box 7240 \\ 53072 Bonn \\ Germany \\ Phone: +49-228-3894-0 \\ Fax: +49-228-3894-180 \\ E-mail: iza@iza.org
}

\begin{abstract}
Any opinions expressed here are those of the author(s) and not those of the institute. Research disseminated by IZA may include views on policy, but the institute itself takes no institutional policy positions.

The Institute for the Study of Labor (IZA) in Bonn is a local and virtual international research center and a place of communication between science, politics and business. IZA is an independent nonprofit company supported by Deutsche Post World Net. The center is associated with the University of Bonn and offers a stimulating research environment through its research networks, research support, and visitors and doctoral programs. IZA engages in (i) original and internationally competitive research in all fields of labor economics, (ii) development of policy concepts, and (iii) dissemination of research results and concepts to the interested public.
\end{abstract}

IZA Discussion Papers often represent preliminary work and are circulated to encourage discussion. Citation of such a paper should account for its provisional character. A revised version may be available directly from the author. 
IZA Discussion Paper No. 2324

September 2006

\section{ABSTRACT \\ Identification of Peer Effects Using Group Size Variation}

This paper considers the semiparametric identification of endogenous and exogenous peer effects based on group size variation. We show that Lee (2006)'s linear-in-means model is generically identified, even when all members of the group are not observed. While unnecessary in general, homoskedasticity may be required in special cases to recover all parameters. Extensions to asymmetric responses to peers and binary outcomes are also considered. Once more, most parameters are semiparametrically identified under weak conditions. However, recovering all of them requires more stringent assumptions. Eventually, we bring theoretical evidence that the model is more adapted to small groups.

JEL Classification: $\quad$ C14, C21, C25

Keywords: social interactions, linear-in-means model, semiparametric identification

Corresponding author:

Denis Fougère

CREST-INSEE

15, Boulevard Gabriel Péri

92245 Malakoff Cedex

France

E-mail: fougere@ensae.fr 


\section{Introduction}

In a seminal paper, Manski (1993) showed that in a linear-in-expectations model with social interactions, endogenous and exogenous peer effects cannot be identified separately. To overcome this negative result, empirical studies usually rely on instrumental strategies, which impose strong exogeneity conditions. In the context of pupils achievement for instance ${ }^{1}$, Hoxby (2000) and Ammermueller and Pischke (2006) suppose that variations in time or between classrooms within the same school are random. Moreover, estimation is based upon reduced forms, so that the structural parameters cannot be recovered. In other terms, one cannot distinguish between exogenous and endogenous peer effects. However, Lee (2006) has recently proposed a modified version of the social interaction model, which corresponds to a linear-in-means model, and which is shown to be identifiable without any of the previous restrictive assumptions, thanks to the group size variation.

Whereas Lee (2006) is mainly concerned with the estimation of the linear-in-means model, we focus on its identification. More precisely, the aim of our paper is threefold. Firstly, we clarify the identification result proposed by Lee (2006) for the linear-in means model. We show that the crucial assumptions here are 1) the knowledge of the group sizes, and 2) the fact that group sizes take at least three different values. On the other hand, and contrary to usual identification strategies based on reduced forms and exclusion restrictions, one does not need to observe all members of the group. This contrasts with the method based on the reduced form, where measurement errors arise as soon as some members of the groups are missing in the data (see Graham and Hahn, 2005). Moreover, neither parametric assumption nor homoskedasticity restriction (as assumption 1 in Lee's paper, 2006) is needed in general. Yet, we show that, in some special cases, the homoskedasticity assumption is required for recovering the structural parameters.

Secondly, we extend the analysis beyond the linear-in-means model. One important limitation of this model is the fact that the mean outcome does not depend on the allocation of individuals across groups. We consider a model with asymmetric responses to peers, and show that almost all parameters can then be identified. However, some parameters are identified only when the whole group is observed. We also consider the case of binary outcomes. Iden-

\footnotetext{
${ }^{1}$ In the following, we will often consider the example of peer effects at school, although the model could also be applied to other topics like smoking (see e.g. Krauth, 2006), productivity in teams (see Rees et al., 2003) or retirement (Duflo and Saez, 2002).
} 
tification of discrete models with social interactions has already been studied by, e.g., Brock and Durlauf (2001, 2004). Our model is slightly different, though, as we assume that social interactions may affect individuals through peers' latent variables rather than through their observable outcomes. This is convenient when only binary outcomes are observable, because of data limitation. To the best of our knowledge, this is the first time such a model is studied in the literature. The attractive feature of our result is that it does not rely on any functional assumption concerning the errors. Yet, due to data limitation, homoskedasticity is needed to recover endogenous peer effects.

Thirdly, we discuss the theoretical background of the linear-in-means model. We show that, in the framework of a noncooperative game between members of the group, the Nash equilibrium satisfies Lee's model (2006), Manski's model (1993) or an intermediate version of these two models, depending on the amount of common knowledge of the players. Lee's model (2006) arises when their information is rich whereas Manski's model emerges when players have little information on their peers. This, along with the slow rate of convergence obtained by Lee (2006) when groups are large, shows that our model should rather be used when groups are small. Lastly, we also show that our model can be viewed as the stationary equilibrium of a dynamic model. Interestingly, one of the identifying assumption arises as a stability condition of this model.

The paper is organized as follows. The first section goes back on the identification of the linear-in-means model. Section two considers an extension to the case of asymmetric responses to peers. Section three is devoted to the discrete case. The fourth section discusses the theoretical foundations of the linear-in-means model. Section five concludes. Proofs are given in the appendix.

\section{The linear-in-means model}

Suppose that we observe $R$ non-overlapping groups $(r=1, \ldots, R)$. Group $r$ has size $m_{r}$. The outcome variable $y_{r i}$ for individual $i$ in group $r$ is assumed to be a linear function of her own observable covariates, denoted $x_{r i}$, but also of the outcome variables and observable covariates of her peers, and of a group-specific (fixed) effect, according to the linear-in-means model of 
Lee $(2006):^{2}$

$$
y_{r i}=x_{r i} \beta_{10}+\left(\frac{1}{m_{r}-1} \sum_{j=1, j \neq i}^{m_{r}} y_{r j}\right) \lambda_{0}+\left(\frac{1}{m_{r}-1} \sum_{j=1, j \neq i}^{m_{r}} x_{r j}\right) \beta_{20}+\alpha_{r}+\varepsilon_{r i} .
$$

Following the terminology introduced by Manski (1993), the second term in the right hand side corresponds to the endogenous peer effect, the third refers to the exogenous peer effects and $\alpha_{r}$ is a contextual (group-specific) effect. This model essentially departs from the one considered by Manski (1993) or by Graham and Hahn (2005) by replacing, on the right-hand side, the expectations relative to the whole group by the means of outcomes and covariates in the group of peers. ${ }^{3}$ As shown by Lee (2006), this small difference allows to obtain identification of the structural parameters, as soon as there is sufficient group variation.

In this section, we clarify and extend some results obtained by Lee (2006). We restrict our analysis to the case where $m_{r}$ does not depend on the size of the sample. ${ }^{4}$ We believe that, in practice, such an assumption is virtually always satisfied. For instance, there is no reason why the mean classroom size should depend on the size of the sample. Moreover, this restriction enables us to clarify the identification results by showing what comes from the usual exogeneity condition (see assumption A5 below) and when homoscedasticity is necessary (see theorem 2 below). On the other hand, we do not impose that all members of the groups are observed; we denote $n_{r}$ the number of sampled individuals in group $r$.

As emphasized by Lee (2006), identification is achieved by the within-group equation, that is (see equation (2.5) of Lee, 2006)

$$
W_{n_{r}} \widetilde{Y}_{r}=W_{n_{r}} \widetilde{X}_{r}\left(\frac{\left(m_{r}-1\right) \beta_{10}-\beta_{20}}{m_{r}-1+\lambda_{0}}\right)+W_{n_{r}} \frac{\widetilde{\varepsilon}_{r}}{1+\lambda_{0} /\left(m_{r}-1\right)},
$$

where $\widetilde{Y}_{r}$ (respectively, $\widetilde{X}_{r}$ and $\widetilde{\varepsilon}_{r}$ ) is the vector of outcomes (respectively, of observed covariates and unobserved residuals) for individuals sampled in group $r$, and $W_{n_{r}}$ denotes the within-group matrix of size $n_{r}$. To recover the structural parameters, we use the variation in the slope coefficient $\beta(m)=\left((m-1) \beta_{10}-\beta_{20}\right) /\left(m-1+\lambda_{0}\right)$. For this purpose, we make the following assumptions:

\footnotetext{
${ }^{2}$ On the contrary to Lee (2006), we do not distinguish $x_{r i, 1}$ from $x_{r i, 2}$. This is without loss of generality since it is sufficient to set $x_{r i}=\left(x_{r i, 1}, x_{r i, 2}\right)$ and to constraint some of the parameters to be zero to obtain his model. Otherwise, we use Lee's notations for facilitating comparison with his paper.

${ }^{3}$ Graham and Hahn (2005) makes the further restriction that $\beta_{20}=0$, i.e. that there are no exogenous peer effects.

${ }^{4}$ This is approximately the scenario with small group interactions of Lee (2006).
} 
A1. For all $r=1, \ldots, R,\left(\widetilde{Y}_{r}, \widetilde{X}_{r}, m_{r}, n_{r}\right)$ are i.i.d.

A2. $\sup \left(\operatorname{Supp}\left(n_{1}\right)\right) \geq 2\left(\right.$ where $\left.\operatorname{Supp}\left(n_{1}\right)=\left\{k \in \mathbb{N}, \operatorname{Pr}\left(n_{1}=k\right)>0\right\}\right)$.

A3. $m_{1}, \ldots, m_{r}$ are known and $\operatorname{card}\left(\operatorname{Supp}\left(m_{1}\right)\right) \geq 3$ (where for all $A \subset \mathbb{N}, \operatorname{card}(A)=$ $\left.\sum_{i=1}^{+\infty} 1\{i \in A\}\right)$.

A4. For all $1 \leq i, j \leq m_{1}, E\left[x_{1 i}^{\prime} \varepsilon_{1 j} \mid m_{1}, n_{1}\right]=0$.

A5. $E\left[\widetilde{X}_{1}^{\prime} W_{n_{r}} \widetilde{X}_{1} \mid m_{1}, n_{1}\right]$ is almost surely nonsingular.

A6. $\lambda_{0}>1-\min \left(\operatorname{Supp}\left(m_{1}\right)\right)$.

Assumption A2 simply states that the within-group approach is possible. Assumption A3 is crucial; it imposes that group sizes are known and that these sizes vary sufficiently in the sample. Assumption A1, A4 and A5 are standard in linear panel data models, except that conditional expectations depend here both on the number of observed individuals in each group and on the group size. Conditioning by $n_{1}$ does not cause any trouble if, for instance, the observed individuals are drawn at random from the group. Finally, assumption A6, which is very similar to assumption 4 introduced by Lee (2006), ensures that $\beta(m)$ exists for all $m \in \operatorname{Supp}\left(m_{1}\right){ }^{5}$ As we will see in section 5 , this assumption arises as the stability condition of a dynamic model.

Theorem 1. Under assumptions A1-A6, $\beta_{10}$ is identified. Moreover,

- if $\beta_{20} \neq-\lambda_{0} \beta_{10}$, then $\lambda_{0}$ and $\beta_{20}$ are identified;

- if $\beta_{20}=-\lambda_{0} \beta_{10}$, then $\lambda_{0}$ is not identified and $\beta_{20}$ is identified up to a constant.

This theorem states that all parameters are generally identified provided that there is sufficient variation in the group sizes, $\left(m_{r}\right)_{r}$. As a notable exception, identification is lost in the absence of endogenous and exogenous peer effects (since then $\beta_{20}=-\lambda_{0} \beta_{10}=0$ ). One can always rationalize such a model with any $\lambda_{0}^{\prime} \neq 0$ and $\beta_{20}^{\prime}=-\lambda_{0}^{\prime} \beta_{10}$. Below, we provide a method which yields identification in this case, but it relies on a stronger assumption of homoskedasticity. In any case, one can check whether identification is lost or not, since this amounts to test whether $\beta($.$) , which is always identified, is constant.$

\footnotetext{
${ }^{5}$ This assumption is not minimal here, since $\lambda_{0} \notin-\operatorname{Supp}\left(m_{1}-1\right)$ would be sufficient for this purpose. But it will be necessary in this form in theorems 2 and 5 .
} 
Contrary to the reduced form approach, we do not need to know the means $\left(\bar{x}_{r}\right)_{1 \leq r \leq R}$ on the whole groups to identify the parameters. Thus the problem of measurement error of $\bar{x}_{r}$, which appears when some individuals in the group are unobserved, does not arise in our framework. Here the crucial assumption is the knowledge of the group size. If it is unknown but can be estimated, the measurement error problem comes back in a nonlinear way. The issue of identification in this case is left for future research. ${ }^{6}$

Another identifying assumption is the additive nature of the group size effect. Indeed, $m_{r}$ may be correlated with $\alpha_{r}$ in a general way, but we cannot add interaction terms $m_{r} \times x_{r i}$ to the list of regressors, since then assumption A5 would fail. Moreover, $\beta_{10}, \beta_{20}$ and $\lambda_{0}$ are assumed to be independent of the group size. An informal test of this assumption would be to estimate $\left(\beta_{10}, \beta_{20}, \lambda_{0}\right)$ on strata made of groups with at least three different sizes, and to compare the estimates obtained from different strata.

If $\beta_{20}=-\lambda_{0} \beta_{10}$, then $\lambda_{0}$ and $\beta_{20}$ cannot be identified. However they can be recovered by studying variance variation under an homoskedasticity condition (assumption A7 below). More precisely, the conditional variance of the residuals should not depend on the group size. This hypothesis is quite weak since it does not restrict the relationship between the residuals $\varepsilon_{r i}$ and the covariates $x_{r i}$. Moreover, under A7, one needs less variation on the group sizes than previously and we can replace assumption A3 by A3'.

A3'. $m_{1}, \ldots, m_{R}$ are i.i.d. and known; $\operatorname{card}\left(\operatorname{Supp}\left(m_{1}\right)\right) \geq 2$.

A7. $\operatorname{Var}\left(\widetilde{\varepsilon_{1}} \mid n_{1}, m_{1}\right)=\sigma^{2} I_{n_{1}}$ where $I_{n_{1}}$ is the identity matrix of size $n_{1}$.

Theorem 2. Under assumptions A1-A2, A3' and A4-A7, $\left(\beta_{10}, \lambda_{0}, \beta_{20}\right)$ are identified.

The idea of using second order moments has already been used by Graham (2005) to identify peer effects. On the contrary to us, however, he can only estimate the posterior distribution of $\lambda_{0}$ in a Bayesian framework.

\footnotetext{
${ }^{6}$ Following Schennach (2004), the model would still be identified if two independent measures of $m_{r}$ were available. The remaining issue is whether the model is identified with only one measure, as it is (under weak conditions) in a linear model (see, e.g., Lewbel, 1997).
} 


\section{Asymmetric responses to peers' outcomes}

One major limitation of the basic linear-in-means model is its functional form. In particular, the fact that everybody reacts similarly to peers is often considered implausible. ${ }^{7}$ Moreover, this restriction implies that the composition of groups does not affect the mean outcome. Hence, if this outcome corresponds to the individual utility, rearranging groups is useless in terms of public policy under a utilitarian social welfare. ${ }^{8}$ For instance, losses due to the reallocation of students in classrooms should perfectly compensate for the gains of these reallocation. Thus, the model implies that the much debated issue of tracking versus mixing in classrooms is irrelevant in terms of efficiency.

To allow for asymmetric reactions to endogenous effects, one could consider, as Cooley (2006), a general nonlinear model. However, the identification of her model relies on the existence of a valid instrument. Instead, we propose a simple extension to the basic model (1) that does not require any exclusion restriction, because our identification strategy still works out in this framework. More precisely, we suppose that

$$
y_{r i}=x_{r i} \beta_{10}+\left(\frac{1}{m_{r}-1} \sum_{j=1, j \neq i}^{m_{r}} y_{r j}\right) \lambda_{0}\left(t_{r i}\right)+\left(\frac{1}{m_{r}-1} \sum_{j=1, j \neq i}^{m_{r}} x_{r j}\right) \beta_{20}\left(t_{r i}\right)+\alpha_{r}+\varepsilon_{r i},
$$

where $t_{r i}$ denotes an observable characteristic of individual $i$. For the sake of simplicity, we consider that $t_{r i}$ is binary (and may take value 0 or 1 ). ${ }^{9}$ The assumption that $\beta_{10}$ does not depend on $t_{r i}$ can be relaxed without loss of generality by including in $x_{r i}$ some interaction terms between $t_{r i}$ and the other covariates. On the other hand, an exclusion restriction is needed for identification, and we assume that $x_{r i}$ is not reduced to $t_{r i}$. Let us denote $x_{r i}^{-t}$ the components of $x_{r i}$ different from $t_{r i}$.

Though simple, this model enables us to overcome the aforementioned drawback. Indeed,

\footnotetext{
${ }^{7}$ For instance, inside the classroom, the race and gender composition variables could interact with individual race and gender. Hoxby (2000), Angrist and Lang (2004), and Cooley (2006) find that such interactions are important.

${ }^{8}$ However, under a social welfare function with inequality aversion, the composition of the groups does in general matter in the basic linear-in-model model.

${ }^{9}$ The extension to any discrete variable is straightforward. On the other hand, the case of a continuous variable is more tedious and not considered here.
} 
some tedious algebra gives:

$$
\begin{aligned}
\bar{y}_{r}= & \frac{1}{1-\left(\frac{m_{r}(0) \lambda_{0}(0)}{m_{r}-1+\lambda_{0}(0)}+\frac{m_{r}(1) \lambda_{0}(1)}{m_{r}-1+\lambda_{0}(1)}\right)} \\
& \times\left[\left(\frac{m_{r}(0)}{m_{r}\left(1+\frac{\lambda_{0}(0)}{m_{r}-1}\right)} \bar{x}_{r}(0)\left(\beta_{10}-\frac{\beta_{20}(0)}{m_{r}-1}\right)+\frac{m_{r}(1)}{m_{r}\left(1+\frac{\lambda_{0}(1)}{m_{r}-1}\right)} \bar{x}_{r}(1)\left(\beta_{10}-\frac{\beta_{20}(1)}{m_{r}-1}\right)\right)\right. \\
& \left.+\left(\frac{m_{r}(0) \beta_{20}(0)}{m_{r}-1+\lambda_{0}(0)}+\frac{m_{r}(1) \beta_{20}(1)}{m_{r}-1+\lambda_{0}(1)}\right) \bar{x}_{i}+\frac{m_{r}(0)\left(\alpha_{r}+\bar{\varepsilon}_{r}(0)\right)}{m_{r}\left(1+\frac{\lambda_{0}(0)}{m_{r}-1}\right)}+\frac{m_{r}(1)\left(\alpha_{r}+\bar{\varepsilon}_{r}(1)\right)}{m_{r}\left(1+\frac{\lambda_{0}(1)}{m_{r}-1}\right)}\right],
\end{aligned}
$$

where, for $k \in\{0,1\}, \bar{x}_{r}(k)$ denotes the mean among individuals $i$ of group $r$ for whom $t_{r i}=k$, and where $m_{r}(k)$ is the size of this subgroup. Contrary to the basic model, it is now possible to increase the average outcome of the population, $\bar{y}=\frac{1}{m} \sum_{r=1}^{R} m_{r} \bar{y}_{r}$, by changing the $\left(m_{r}(k)\right)_{1 \leq r \leq R}$.

Let $\beta_{10}^{t}$ (respectively, $\left.\beta_{20}^{t}(t)\right)$ denote the component of the parameter vector $\beta_{10}$ (respectively, $\left.\beta_{20}(t)\right)$ which corresponds to covariate $t$. For instance, if $t$ denotes the ethnic group of a pupil in a classroom (with, e.g., $t=0$ for a white pupil and $t=1$ for a black pupil), $\beta_{10}^{t}$ denotes the impact of being black on, e.g., achievement, and $\beta_{20}^{t}(0)$ denotes the effect of the proportion of blacks in the group on the achievement of a white pupil. $\beta_{10}^{-t}$ and $\beta_{20}^{-t}(t)$ correspond to the other components of parameter vectors $\beta_{10}$ and $\beta_{20}(t)$. The following additional assumptions are required for identification of these parameters:

A2'. $\sup \left(\operatorname{Supp}\left(n_{1}(k)\right)\right) \geq 2$ for $k \in\{0,1\}$.

A5'. $E\left[\widetilde{X}_{1}^{\prime-t}(k) W_{n_{r}(k)} \widetilde{X}_{1}^{-t}(k) \mid m_{1}, n_{1}\right]$ (where $\widetilde{X}_{1}^{-t}(k)$ is the subvector of $\widetilde{X}_{1}^{-t}$ for individuals $i$ such as $t_{i}=k$ ) is almost surely nonsingular for $k \in\{0,1\}$.

A8. $\beta_{20}(k) \neq-\lambda_{0}(k) \beta_{10}$ for $k \in\{0,1\}$.

A9. $\left(\bar{x}_{r}, \bar{y}_{r}\right)_{1 \leq r \leq R}$ are known.

Assumption A2' states that there are some subgroups in which at least two individuals are observed, so that we can use the within equation on subgroups with a positive probability. Assumption A5' supposes that variables are linearly independent within subgroups, almost surely. Assumption A8 is made for convenience. If it does not hold, one can still obtain partial identification results, as in theorem 1. Assumption A9 is restrictive, since it supposes in practice that all individuals belonging to a group are observed. However, it is not required to identify parameters $\beta_{10}^{-t}, \lambda_{0}(k)$ and $\beta_{20}^{-t}(k), k \in\{0,1\}$. 
Theorem 3. Under assumptions A1, A2', A3, A4, A5', A6 and A8, $\beta_{10}^{-t}, \lambda_{0}(k)$ and $\beta_{20}^{-t}(k)(k \in$ $\{0,1\})$ are identified. Moreover, if assumption A9 holds, $\beta_{10}^{t}$ and $\beta_{20}^{t}(k)(k \in\{0,1\})$ can also be recovered.

\section{The discrete case}

In this section, we investigate whether the parameters are still identified when one cannot observe directly the outcome variable but only a rough (binary) measure of it. In other terms, we observe $y_{r i}=1\left\{y_{r i}^{*} \geq 0\right\}$, where $y_{r i}^{*}$ satisfies equation (1). For instance, to study pupil achievement, only grade retention rather than test scores may be available. Similarly, in violence studies, only criminal (that is, sufficiently violent) acts can be observed by the econometrician. Note that these models remain essentially linear because the underlying model is linear. One could also study the case where $y_{r i}^{*}$ depends on $y_{r j}$ rather than $y_{r j}^{*}$. Such models, which have been studied by Brock and Durlauf (2001, 2004), Bayer and Timmins (2002), Tamer (2003) and Krauth (2006), are more complex because in general multiple equilibria arise.

When the outcome is a binary variable, the reduced-form equation (3) is useless for identification because $W_{n_{r}} \widetilde{Y}_{r}^{*}$ (where $\widetilde{Y}_{r}^{*}$ is the vector of latent outcomes for observed individuals of $r$ ) has no observational counterpart. Instead, we rely on equation (4) below. The result is obtained under A6', which is slightly stronger than assumption A6. Note that A6' holds under the stability condition of the dynamic model that will be proposed in section 5 (see proposition 1 below).

A6': $\lambda_{0}>1-\min \left(\operatorname{Supp}\left(m_{1}\right)\right)$ and $\lambda_{0} \neq 1$.

Lemma 1. Suppose that $y_{r i}=1\left\{y_{r i}^{*} \geq 0\right\}$ with $y_{r i}^{*}$ satisfying equation (1), and that assumption A6' holds. Then the model is observationally equivalent to

$$
\begin{aligned}
y_{r i}=1 & \left\{x_{r i}\left(\beta_{10}-\frac{\beta_{20}}{m_{r}-1}\right)\right. \\
& +\left[\overline{x_{r}} \frac{m_{r}}{m_{r}-1}\left(\beta_{20}+\frac{\beta_{10}+\beta_{20}}{1-\lambda_{0}} \lambda_{0}\right)+\alpha_{r}\left(1+\frac{m_{r}}{m_{r}-1} \frac{\lambda_{0}}{1-\lambda_{0}}\right)\right] \\
& \left.+\overline{\varepsilon_{r}} \frac{m_{r}}{m_{r}-1} \frac{\lambda_{0}}{1-\lambda_{0}}+\varepsilon_{r i} \geq 0\right\} .
\end{aligned}
$$


The term in brackets corresponds to a group-specific effect. Thus we are led back to a binary model for panel data. Identification of such a model has been considered, among others, by Manski (1987), and our analysis relies on his paper. The group indices are omitted for the ease of notation. Then $x_{j}^{k}$ denotes the $k$-th covariate of individual $j$. The following assumptions are needed for identification:

A10. $\left(\varepsilon_{1}, \ldots, \varepsilon_{m}\right)$ are exchangeable conditionally on $\left(m, x_{1}, \ldots, x_{m}, \alpha\right)$. The support of

$$
F_{\varepsilon_{1}+\bar{\varepsilon} \frac{m}{m-1} \frac{\lambda_{0}}{1-\lambda_{0}} \mid m, x_{1}, \ldots, x_{m}, \alpha}\left(. \mid m^{*}, x_{1}^{*}, \ldots, x_{m}^{*}, \alpha^{*}\right)
$$

(where, for any couple of random variables $(u, v), F_{u \mid v}$ denotes the conditional c.d.f. of $u$ given $v)$ is $\mathbb{R}$ for all $\left(m^{*}, x_{1}^{*}, \ldots, x_{m}^{*}, \alpha^{*}\right)$.

A11. Let $z=x_{2}-x_{1} \cdot{ }^{10}$ The support of $F_{z}$ is not contained in any proper linear subspace of $\mathbb{R}^{K}$ (where $K$ is the dimension of $x_{r i}$ ).

A12. There exists $k_{0}$ such that $z^{k_{0}}$ has everywhere a positive Lebesgue density conditional on $\left(m, z^{1}, \ldots, z^{k_{0}-1}, z^{k_{0}+1}, \ldots, z^{K}\right)$ and $\beta_{10}^{k_{0}}=1$. Without loss of generality we set $k_{0}=1$.

The first part of assumption A10 holds for instance if $\left(\varepsilon_{i}\right)_{i=1, \ldots, m}$ is conditionally independent of $\left(x_{i}\right)_{i=1, \ldots, m}$ given $m$ and $\alpha$, and if $\left(\varepsilon_{i}\right)_{i=1, \ldots, m}$ are exchangeable conditionally on $m, \alpha$. Hence, it is satisfied if the $\left(\varepsilon_{i}\right)_{i=1, \ldots, m}$ are i.i.d. and independent of $\left(x_{1}, \ldots, x_{m}, m, \alpha\right)$. The second part of assumption A10 is a technical condition, which is identical to the second part of assumption 1 set forth by Manski (1987). Assumption A11 ensures that $z$ varies enough within a group. As usually in binary models, one parameter must be normalized and this is the purpose of A12. However, a small difficulty arises here, because the reduced form does not allow us to recover the sign of the structural parameters. A sufficient condition is to fix one parameter (and not only its absolute value): thus we set $\beta_{10}^{1}=1 .^{11}$

Theorem 4. Suppose that assumptions A1-A3, A6' and A10-A12 hold. Then $\beta_{10}$ is identified. Moreover,

- if $\beta_{20} \neq \beta_{20}^{1} \beta_{10}$, then $\beta_{20}$ is identified,

- if $\beta_{20}=\beta_{20}^{1} \beta_{10}, \beta_{20}^{1}$ is not identified and the other $\beta_{20}^{k}$ are identified up to $\beta_{20}^{1}$.

On the other hand, $\lambda_{0}$ is not identified.

\footnotetext{
${ }^{10}$ Without loss of generality, we assume here that individuals 1 and 2 are observed.

${ }^{11}$ Obviously, theorem 5 also holds with $\beta_{10}^{1}=-1$.
} 
If fewer parameters than in model (1) are identified, theorem 4 shows that the main attractive features of the method remain. Without any exclusion restriction and even if only two members of the groups are observed, $\beta_{10}$ and $\beta_{20}$ are generally identified. Similarly to the result of theorem 1, identification of $\beta_{20}$ is lost when there is no exogenous effect, because in this case $\beta_{20}=\beta_{20}^{1} \beta_{10}=0$. That $\lambda_{0}$ cannot be recovered is not surprising as this parameter only appears in the fixed effect and the residuals (see equation (4)). Heuristically, without any hypothesis imposed on these terms, any $\lambda_{0}$ can be rationalized by changing accordingly $\alpha$ and the $\left(\varepsilon_{i}\right)_{1 \leq i \leq m}$.

Thus, stronger assumptions are needed for identifying $\lambda_{0}$. One possibility is to observe $\bar{x}$ and to restrict the dependence between the residuals and the covariates.

A9'. $\bar{x}$ is observed.

A13. $\left(\varepsilon_{1}, \ldots, \varepsilon_{m}, \alpha\right) \Perp\left(x_{1}, \ldots, x_{m}\right) \mid m, \bar{x}$.

A14. $\operatorname{Var}\left(\varepsilon_{1}, \ldots, \varepsilon_{m}, \alpha \mid \bar{x}, m\right)=\left(\begin{array}{cc}\operatorname{Var}\left(\varepsilon_{1} \mid \bar{x}\right) I_{m} & 0 \\ 0 & \operatorname{Var}(\alpha \mid \bar{x})\end{array}\right)$.

A15. Given $(\bar{x}, m)$, the support of $\left\{x_{1}\left(\beta_{10}-\frac{\beta_{20}}{m-1}\right), x_{2}\left(\beta_{10}-\frac{\beta_{20}}{m-1}\right)\right\}$ is $\mathbb{R}^{2}$.

As mentioned above, assumption A9' is a restrictive condition as it imposes either to observe all individuals in the group or to consider only the covariates for which the means are known. Assumption A13 is in the same spirit than assumption A10. It restricts the dependence between $\alpha$ and the covariates to a dependence on the mean. Assumption A14 is the assumption of homoskedasticity in $m$; it is very similar to assumption A7. The difference between both assumptions stems from the identifying equation we use in both cases. In the discrete model, $\alpha$ remains in expression (4) and thus its variance must be modeled as well as its covariance with the $\left(\varepsilon_{i}\right)_{1 \leq i \leq m} \cdot{ }^{12}$ Lastly, assumption A15 is a condition of large support. It especially implies that $m \geq 3$. Otherwise, indeed, the two variables belong to a line in $\mathbb{R}^{2}$.

Theorem 5. Under assumptions A1-A3, A6', A9', and A10-A15 and if $\beta_{20} \neq \beta_{20}^{1} \beta_{10}, \lambda_{0}$ is also identified.

\footnotetext{
${ }^{12}$ The assumption of no covariance is not restrictive. Indeed, if the correlation between $\varepsilon_{i}$ and $\alpha$ is not zero and independent of $i$, we can always reparametrize the model in order to make them uncorrelated.
} 


\section{Theoretical background}

Model (1) is very close to the one considered by Manski (1993) or by Graham and Hahn (2005). Yet conclusions much differ since the basic linear-in-means model is generically identified without any exclusion restriction. Thus, one should set forth the reasons to choose this model rather than the linear-in-expectations model. An argument borrowed from game theory shows that this choice is in fact better justified for small groups. Indeed, we show below that identification depends on the information set of players. Equation (1) arises when players are fully informed, whereas Manski's model corresponds to an imperfect information situation. We also consider an intermediate situation where homoscedasticity is lost, and thus theorem 2 cannot be applied.

Suppose that the utility of player $i$ has the following form:

$$
\begin{aligned}
\mathcal{U}_{r i}\left(e_{r i},\left(e_{r j}\right)_{j \neq i}\right)= & e_{r i}\left[x_{r i} \beta_{10}+\left(\frac{1}{m_{r}-1} \sum_{j=1, j \neq i}^{m_{r}} e_{r j}\right) \lambda_{0}\right. \\
& \left.+\left(\frac{1}{m_{r}-1} \sum_{j=1, j \neq i}^{m_{r}} x_{r j}\right) \beta_{20}+\alpha_{r}+\varepsilon_{r i}\right]-\frac{1}{2} e_{r i}^{2},
\end{aligned}
$$

where we suppose here that the individual specific terms $\left(\varepsilon_{r i}\right)_{i}$ are mutually independent and independent of $\left(x_{r i}, \alpha_{r}, m_{r}\right)$. In this game, the marginal returns of individual $i$ depend on her peers' outcomes. This captures the fact that people are influenced by their peers' behavior. If $\lambda_{0}>0$, player $i$ tries to conform to other members of her group, whereas she tries to stand out from them when $\lambda_{0}<0$. This model is close to the one developed by Cooley (2006) for examining pupil achievement in the classroom.

Assuming that $\alpha_{r}$ and the $\left(x_{r i}, \varepsilon_{r i}\right)_{1 \leq i \leq m_{r}}$ are observed by all players in group $r$, the Nash equilibrium of the game $\left(e_{r 1}^{*}, \ldots, e_{r m_{r}}^{*}\right)$ satisfies equation (1). Thus, using theorems 1 and 2 , all parameters are identified provided that there is sufficient variation in group sizes. ${ }^{13}$

Now suppose that the $\left(\varepsilon_{r j}\right)_{j \neq i}$ are unobserved by player $i$. This situation is realistic in moderately large groups where each player observes the characteristic $x_{r j}$ of $j$ but not her

\footnotetext{
${ }^{13}$ This conclusion still holds if we do not observe the optimal effort $e_{r i}^{*}$ but instead the corresponding outcome $y_{r i}=e_{r i}^{*}+\eta_{r i}$, where $\eta_{r i}$ is an unanticipated shock, independent of other variables. A quick examination reveals that in the within-group equation (3), shocks are not homoskedastic anymore, but the proof of theorem 2 can be adapted provided that at least three different group sizes are available.
} 
idiosyncratic shock. Then the Bayesian Nash equilibrium satisfies: ${ }^{14}$

$$
e_{r i}^{*}=x_{r i} \beta_{10}+\left(\frac{1}{m_{r}-1} \sum_{j=1, j \neq i}^{m_{r}} E\left(e_{r j}^{*} \mid \varepsilon_{r i}\right)\right) \lambda_{0}+\left(\frac{1}{m_{r}-1} \sum_{j=1, j \neq i}^{m_{r}} x_{r j}\right) \beta_{20}+\alpha_{r}+\varepsilon_{r i} .
$$

Taking the conditional expectation leads to, for $i \neq k$,

$$
E\left(e_{r i}^{*} \mid \varepsilon_{r k}\right)=x_{r i} \beta_{10}+\left(\frac{1}{m_{r}-1} \sum_{j=1, j \neq i}^{m_{r}} E\left(e_{r j}^{*}\right)\right) \lambda_{0}+\left(\frac{1}{m_{r}-1} \sum_{j=1, j \neq i}^{m_{r}} x_{r j}\right) \beta_{20}+\alpha_{r},
$$

because, by independence of the $\left(\varepsilon_{r i}\right)_{i}, E\left[E\left(e_{r j}^{*} \mid \varepsilon_{r i}\right) \mid \varepsilon_{r k}\right]=E\left[E\left(e_{r j}^{*} \mid \varepsilon_{r i}\right)\right]=E\left(e_{r j}^{*}\right)$. Hence, taking the expectation of (5) and comparing with (6) leads to $E\left(e_{r i}^{*} \mid \varepsilon_{r k}\right)=E\left(e_{r i}^{*}\right)$ for all $i \neq k$. Replacing $E\left(e_{r j}^{*} \mid \varepsilon_{r i}\right)$ by $E\left(e_{r j}^{*}\right)$ in (5), we get $e_{r i}^{*}=E\left(e_{r i}^{*}\right)+\varepsilon_{r i}$. Finally, substituting $e_{r j}^{*}-\varepsilon_{r j}^{*}$ to $E\left(e_{r j}^{*}\right)$ in $(5)$, we obtain:

$$
\begin{aligned}
e_{r i}^{*}= & x_{r i} \beta_{10}+\left(\frac{1}{m_{r}-1} \sum_{j=1, j \neq i}^{m_{r}} e_{r j}^{*}\right) \lambda_{0}+\left(\frac{1}{m_{r}-1} \sum_{j=1, j \neq i}^{m_{r}} x_{r j}\right) \beta_{20}+\alpha_{r}-\frac{m_{r}}{m_{r}-1} \overline{\varepsilon_{r}} \lambda_{0} \\
& +\varepsilon_{r i}\left(1+\frac{\lambda_{0}}{m_{r}-1}\right)
\end{aligned}
$$

which is equivalent to

$$
e_{r i}^{*}=x_{r i} \beta_{10}+\left(\frac{1}{m_{r}-1} \sum_{j=1, j \neq i}^{m_{r}} e_{r j}^{*}\right) \lambda_{0}+\left(\frac{1}{m_{r}-1} \sum_{j=1, j \neq i}^{m_{r}} x_{r j}\right) \beta_{20}+\alpha_{r}^{\prime}+\varepsilon_{r i}^{\prime},
$$

where $\alpha_{r}^{\prime}=\alpha_{r}-m_{r} /\left(m_{r}-1\right) \overline{\varepsilon_{r}} \lambda_{0}$ and $\varepsilon_{r i}^{\prime}=\varepsilon_{r i}\left(1+\frac{\lambda_{0}}{m_{r}-1}\right)$. This equation is very similar to (1) and the parameters can be identified using the exogeneity of covariates. However, the errors become heteroskedastic. More precisely, the variance of residuals in the within-group equation (2) is no more dependent on $m_{r}$, so that $\lambda_{0}$ cannot be recovered by using this device, as in theorem 2. Identification of $\beta_{20}$ and $\lambda_{0}$ is lost when $\beta_{20}=-\lambda_{0} \beta_{10}$.

Lastly, let us suppose that the $\left(x_{r j}\right)_{j \neq i}$ are also unobserved by $i$ but that $\alpha_{r}$ is still observed. If groups are large, player $i$ may not know $j$ and thus does not observe neither $\varepsilon_{r j}$ nor $x_{r j}$. On the other hand, she may know the general features of the group, represented by $\alpha_{r}$ and $E\left(x_{r 1} \mid \alpha_{r}\right)$. Then, proceeding as previously, we can easily show that

$$
e_{r i}^{*}=x_{r i} \beta_{10}+E\left(x_{r 1} \mid \alpha_{r}\right) \beta_{20}+E\left(e_{r i}^{*} \mid \alpha_{r}\right) \lambda_{0}+\alpha_{r}+\varepsilon_{r i} .
$$

Thus we are led back to Manski's model (1993), which is not identifiable.

\footnotetext{
${ }^{14}$ All expectations are in fact taken conditionally on $\left(x_{r i}\right)_{1 \leq i \leq m_{r}}$ and $\alpha_{r}$. We omit them for the sake of simplicity.
} 
Another structural interpretation of the model is to consider equation (1) as the stationary state of a dynamic model with interactions. Let $y_{r i}^{t}$ denote the outcome of individual $i$ in group $r$ at date $t$, and suppose that the variation in this outcome at date $t$ is generated by the following first-order differential equation:

$$
\frac{d y_{r i}^{t}}{d t}=y_{r i}^{t} \mu+\left(\frac{1}{m_{r}-1} \sum_{j=1, j \neq i}^{m_{r}} y_{r j}^{t}\right) \lambda_{0}+x_{r i} \beta_{10}+\left(\frac{1}{m_{r}-1} \sum_{j=1, j \neq i}^{m_{r}} x_{r j}\right) \beta_{20}+\alpha_{r}+\varepsilon_{r i} .
$$

In other terms, the variation in the $i$-th individual's outcome at date $t$ depends on her current outcome and on her time-constant observable covariates, but also on the mean of her peers' outcomes at date $t$ and on the mean of their time-constant observable covariates. In the example of a classroom, $\lambda_{0}>0$ implies that a pupil will get better test scores when the mean score of her classmates is higher.

Proposition 1. Equation (7) admits a stable solution for all $r=1 . . R$ if and only if

$$
\left.\mu \leq 0 \text { and } \lambda_{0} \in\right] \mu\left\{\min _{r}\left(m_{r}\right)-1\right\},-\mu[.
$$

Provided that $\mu=-1$, this solution is given by equation (1).

Setting $\mu=-1$ is natural in view of identification. Indeed, in the steady state, $\frac{d y_{r i}^{t}}{d t}=0$ so that equation (7) can be normalized without loss of generality. Proposition 1 states that if the dynamic model is true, then $\lambda_{0}$ lies in a known interval. Interestingly, this restriction $\left(\lambda_{0}>1-\min _{r}\left(m_{r}\right)\right)$ is used both in Lee's paper (assumption 3) and in ours (assumption A6 and $\mathrm{A} 6$ ') to identify the model.

\section{Conclusion}

This paper considers identification of linear social interaction models using group size variation. Provided that the size of the group is known and varies sufficiently, endogenous and exogenous effects can be identified without any exclusion restriction. Moreover, the method does not require to observe all members of the group. The result is extended to asymmetric models and binary outcomes. We also show that, from a game theoretic point of view, our analysis is more relevant for small groups such as classrooms. When groups are large, Manski's model (1993) should be more appropriate in terms of players' information. The result is also in line with the weak identification result of Lee (2006) in the case of large groups. 
Our paper has two main limitations. First, the size of the group is assumed to be known. However, as emphasized by Manski (2000), it is often difficult to define groups on an a priori background. This criticism is common to all models of social interactions, but may be especially problematic here. Indeed, ignoring the boundaries of the group leads (among other difficulties) to measurement errors on the group size, which could prevent identification. Second, we do not consider a fully nonparametric regression. The issue of whether group size variation has an identifying power in this general case remains to be settled.

\section{Acknowledgements}

We would like to thank Stéphane Grégoir, Steve Machin, Amine Ouazad, as well as the participants to the First Summer School of "The Economics of Education and Education Policy in Europe" Marie Curie Research Training Network (Padova, 16-18 June 2006) for their useful comments. The usual disclaimer applies.

\section{Appendix}

\section{Theorem 1}

First, under assumption A4, $E\left(\widetilde{X}_{1}^{\prime} W_{n_{1}} \widetilde{\varepsilon}_{1} \mid n_{1}, m_{1}\right)=0$ and thus, by assumption A5, $\beta(m)$ is identified for all $m \in \operatorname{Supp}\left(m_{1}\right)$. We now prove that the knowledge of $m \mapsto \beta(m)$ allows in general to recover the structural parameters.

Let $\left(m_{1}^{*}, m_{2}^{*}\right) \in \operatorname{Supp}\left(m_{1}\right)^{2}$, then

$$
\frac{\left(m_{1}^{*}-1\right) \beta_{10}-\beta_{20}}{m_{1}^{*}-1+\lambda_{0}}=\frac{\left(m_{2}^{*}-1\right) \beta_{10}-\beta_{20}}{m_{2}^{*}-1+\lambda_{0}}
$$

is equivalent to

$$
-\lambda_{0} \beta_{10}\left(\frac{1}{m_{1}^{*}-1}-\frac{1}{m_{2}^{*}-1}\right)=\beta_{20}\left(\frac{1}{m_{1}^{*}-1}-\frac{1}{m_{2}^{*}-1}\right) .
$$

Hence, if $\beta_{20}=-\lambda_{0} \beta_{10}, \beta($.$) is constant, and if not, \beta($.$) is a one-to-one mapping. In the$ first case, $\beta(m)=\beta_{10}$ for all $m$. Thus $\beta_{10}$ is identified, but $\lambda_{0}$ cannot be recovered by $\beta($.$) .$ Because $\beta_{20}=-\lambda_{0} \beta_{10}, \beta_{20}$ is identified up to a constant.

Now suppose that $\beta_{20} \neq-\lambda_{0} \beta_{10}$ and let $\left(m_{0}^{*}, m_{1}^{*}, m_{2}^{*}\right)$ be three different values in $\operatorname{Supp}\left(m_{1}\right)$. We will prove that the knowledge of $\beta\left(m_{0}^{*}\right), \beta\left(m_{1}^{*}\right)$ and $\beta\left(m_{2}^{*}\right)$ permits to recover $\left(\beta_{10}, \lambda_{0}, \beta_{20}\right)$. 
This amounts to show that the system

$$
\left\{\begin{array}{l}
\beta\left(m_{0}^{*}\right) \lambda_{0}-\left(m_{0}^{*}-1\right) \beta_{10}+\beta_{20}=-\beta\left(m_{0}^{*}\right)\left(m_{0}^{*}-1\right) \\
\beta\left(m_{1}^{*}\right) \lambda_{0}-\left(m_{1}^{*}-1\right) \beta_{10}+\beta_{20}=-\beta\left(m_{1}^{*}\right)\left(m_{1}^{*}-1\right) \\
\beta\left(m_{2}^{*}\right) \lambda_{0}-\left(m_{2}^{*}-1\right) \beta_{10}+\beta_{20}=-\beta\left(m_{2}^{*}\right)\left(m_{2}^{*}-1\right)
\end{array}\right.
$$

has a unique solution. Using the matrix form, we can rewrite the system as $A \zeta_{0}=B$ where $\zeta_{0}=\left(\lambda_{0}, \beta_{10}, \beta_{20}\right)^{\prime}$. If $\operatorname{det}(A) \neq 0, \zeta_{0}$ is identified. Suppose that $\operatorname{det}(A)=0$. Then $\operatorname{com}(A)^{\prime} B=0$ where $\operatorname{com}(A)$ denotes the comatrix of $A$. By using the first line of this equation and the expression of $\operatorname{det}(A)$, we get

$$
\left\{\begin{array}{l}
\left(m_{2}^{*}-m_{1}^{*}\right) \beta\left(m_{0}^{*}\right)+\left(m_{0}^{*}-m_{2}^{*}\right) \beta\left(m_{1}^{*}\right)+\left(m_{1}^{*}-m_{0}^{*}\right) \beta\left(m_{2}^{*}\right)=0 \\
\left(m_{0}^{*}-1\right)\left(m_{2}^{*}-m_{1}^{*}\right) \beta\left(m_{0}^{*}\right)+\left(m_{1}^{*}-1\right)\left(m_{0}^{*}-m_{2}^{*}\right) \beta\left(m_{1}^{*}\right)+\left(m_{2}^{*}-1\right)\left(m_{1}^{*}-m_{0}^{*}\right) \beta\left(m_{2}^{*}\right)=0 .
\end{array}\right.
$$

Hence,

$$
\left\{\begin{array}{l}
\left(m_{2}^{*}-m_{1}^{*}\right) \beta\left(m_{0}^{*}\right)=-\left(m_{0}^{*}-m_{2}^{*}\right) \beta\left(m_{1}^{*}\right)-\left(m_{1}^{*}-m_{0}^{*}\right) \beta\left(m_{2}^{*}\right) \\
m_{0}^{*}\left(m_{2}^{*}-m_{1}^{*}\right) \beta\left(m_{0}^{*}\right)+m_{1}^{*}\left(m_{0}^{*}-m_{2}^{*}\right) \beta\left(m_{1}^{*}\right)+m_{2}^{*}\left(m_{1}^{*}-m_{0}^{*}\right) \beta\left(m_{2}^{*}\right)=0 .
\end{array}\right.
$$

Thus,

$$
\left\{\begin{array}{l}
\left(m_{2}^{*}-m_{1}^{*}\right) \beta\left(m_{0}^{*}\right)+\left(m_{0}^{*}-m_{2}^{*}\right) \beta\left(m_{1}^{*}\right)+\left(m_{1}^{*}-m_{0}^{*}\right) \beta\left(m_{2}^{*}\right)=0 \\
\beta\left(m_{1}^{*}\right)\left(m_{0}^{*}-m_{2}^{*}\right)\left(m_{1}^{*}-m_{2}^{*}\right)+\beta\left(m_{0}^{*}\right)\left(m_{2}^{*}-m_{1}^{*}\right)\left(m_{0}^{*}-m_{2}^{*}\right)=0 .
\end{array}\right.
$$

Because $m_{1}^{*} \neq m_{2}^{*}$ and $m_{0}^{*} \neq m_{2}^{*}$, this implies that $\beta\left(m_{1}^{*}\right)=\beta\left(m_{0}^{*}\right)$, which is in contradiction with the fact that $\beta($.$) is a one-to-one mapping. Thus \operatorname{det}(A) \neq 0$ and $\zeta_{0}$ is identified.

\section{Theorem 2}

Because $m \mapsto \beta(m)$ is identified, Var $\left(\frac{W_{n_{1}} \widetilde{\varepsilon}_{1}}{1+\frac{\lambda_{0}}{m_{1}-1}} \mid n_{1}, m_{1}\right)$ is known. Thus, under assumption $\mathrm{A} 7$,

$$
\operatorname{Var}\left(\frac{W_{n_{1}} \widetilde{\varepsilon}_{1}}{1+\frac{\lambda_{0}}{m-1}} \mid n_{1}, m_{1}\right)=\frac{\sigma^{2}}{\left(1+\frac{\lambda_{0}}{m_{1}-1}\right)^{2}} W_{n_{1}}
$$

Hence, for $m_{1}^{*} \neq m_{2}^{*}$,

$$
C \equiv \frac{\left(1+\frac{\lambda_{0}}{m_{1}^{*}-1}\right)^{2}}{\left(1+\frac{\lambda_{0}}{m_{2}^{*}-1}\right)^{2}}
$$


is identified. Under assumption A6, $\left(1+\frac{\lambda_{0}}{m-1}\right)>0$ for all $m \in \operatorname{Supp}\left(m_{1}\right)$. Thus

$$
\left(\frac{\sqrt{C}}{m_{1}^{*}-1}-\frac{1}{m_{2}^{*}-1}\right) \lambda_{0}=1-\sqrt{C} .
$$

It is clear that $\left(\frac{\sqrt{C}}{m_{1}^{*}-1}-\frac{1}{m_{2}^{*}-1}\right) \neq 0$. Otherwise $C=1$ and then $m_{1}^{*}=m_{2}^{*}$, which contradicts the assumption. Thus $\lambda_{0}$ is identified.

Then, because $m \mapsto \beta(m)$ is identified, $\beta_{10}-\frac{\beta_{20}}{m-1}$ is known for all $m \in \operatorname{Supp}\left(m_{1}\right)$. Taking two different values for $m$ permits to recover $\beta_{20}$, and then $\beta_{10}$.

\section{Theorem 3}

Let $i_{1}$ and $i_{2}$ be two individuals in group $r$ such as $t_{r i_{1}}=t_{r i_{2}}=k$. Then

$$
y_{r i_{1}}-y_{r i_{2}}=\left(x_{r i_{1}}-x_{r i_{2}}\right)\left(\frac{\left(m_{r}-1\right) \beta_{10}-\beta_{20}(k)}{m_{r}-1+\lambda_{0}(k)}\right)+\frac{\varepsilon_{r i_{1}}-\varepsilon_{r i_{2}}}{1+\lambda_{0}(k) /\left(m_{r}-1\right)} .
$$

Thus, by application of Theorem 1 on both subgroups, $\beta_{10}^{-t}, \beta_{20}^{-t}(k)$ and $\lambda_{0}(k)$ are identified. To recover $\beta_{10}^{t}$ and $\beta_{20}^{t}(k)$, we start from

$$
\begin{aligned}
y_{r i}\left(m_{r}-1+\right. & \left.\lambda_{0}\left(t_{r i}\right)\right)-x_{r i}^{-t}\left(\left(m_{r}-1\right) \beta_{10}^{-t}-\beta_{20}^{-t}\left(t_{r i}\right)\right)-m_{r} \bar{y}_{r} \lambda_{0}\left(t_{r i}\right)+m_{r} \bar{x}_{r}^{-t} \beta_{20}^{-t}\left(t_{r i}\right) \\
= & t_{r i}\left(\left(m_{r}-1\right) \beta_{10}^{t}-\beta_{20}^{t}\left(t_{r i}\right)\right)+m_{r} \bar{t}_{r} \beta_{20}^{t}\left(t_{r i}\right)+\left(m_{r}-1\right) \alpha_{r}+\left(m_{r}-1\right) \varepsilon_{r i} .
\end{aligned}
$$

Let $\widehat{y}_{r i}$ denote the left hand side of this equation; it is identified thanks to the previous result and to assumption A9. Taking $i_{1}$ and $i_{3}$ such as $t_{r i_{1}}=1, t_{r i_{3}}=0$, we get

$$
\widehat{y}_{r i_{1}}-\widehat{y}_{r i_{3}}=\left(m_{r}-1\right) \beta_{10}^{t}-\beta_{20}^{t}(1)+m_{r} \bar{t}_{r}\left[\beta_{20}^{t}(1)-\beta_{20}^{t}(0)\right]+\left(m_{r}-1\right)\left(\varepsilon_{r i_{1}}-\varepsilon_{r i_{3}}\right) .
$$

This regression (over groups of the same size but with different $\bar{t}_{r}$ ) enables us to recover the constant $\left(m_{r}-1\right) \beta_{10}^{t}-\beta_{20}^{t}(1)$ and $\beta_{20}^{t}(1)-\beta_{20}^{t}(0)$. Then making $m_{r}$ vary allows us to identify $\beta_{10}^{t}$ and $\beta_{20}^{t}(1)$, and thus $\beta_{20}^{t}(0)$.

\section{Lemma 1}

Applying the between-group operator to (1) gives

$$
\overline{y_{r}^{*}}=\overline{x_{r}}\left(\frac{\beta_{10}+\beta_{20}}{1-\lambda_{0}}\right)+\frac{\alpha_{r}}{1-\lambda_{0}}+\frac{\overline{\varepsilon_{r}}}{1-\lambda_{0}},
$$


since $1 /\left(1-\lambda_{0}\right)$ exists according to assumption A6'. Consequently, replacing $\overline{y_{r}^{*}}$ in equation (1), we obtain

$$
\begin{aligned}
y_{r i}^{*}\left(1+\frac{\lambda_{0}}{m_{r}-1}\right)= & x_{r i}\left(\beta_{10}-\frac{\beta_{20}}{m_{r}-1}\right)+\overline{x_{r}} \frac{m_{r}}{m_{r}-1}\left(\beta_{20}+\frac{\beta_{10}+\beta_{20}}{1-\lambda_{0}} \lambda_{0}\right) \\
& +\alpha_{r}\left(1+\frac{m_{r}}{m_{r}-1} \frac{\lambda_{0}}{1-\lambda_{0}}\right)+\overline{\varepsilon_{r}} \frac{m_{r}}{m_{r}-1} \frac{\lambda_{0}}{1-\lambda_{0}}+\varepsilon_{r i} .
\end{aligned}
$$

Note that this equation is equivalent to (1). Now, under assumption A6', $1+\lambda_{0} /\left(m_{r}-1\right)>0$ for all $r$, so that $y_{r i}^{*} \geq 0$ if and only if $y_{r i}^{*}\left(1+\frac{\lambda_{0}}{m_{r}-1}\right) \geq 0$. Thus, under assumption A6', $y_{r i}=1\left\{y_{r i}^{*} \geq 0\right\}$, where $y_{r i}^{*}$ satisfies equation (1), is observationally equivalent to $y_{r i}$ satisfying equation (4).

\section{Theorem 4}

Assumption A10 implies that the conditional distribution of $\bar{\varepsilon} \frac{m}{m-1} \frac{\lambda_{0}}{1-\lambda_{0}}+\varepsilon_{i}$ is identical for every $i$. Thus assumption 1 in Manski (1987) is satisfied and, using A11 and A12, we can apply directly his result to recover $\frac{(m-1) \beta_{10}-\beta_{20}}{\left|m-1-\beta_{20}^{1}\right|}$. The first term of the vector, $\frac{(m-1) \beta_{10}^{1}-\beta_{20}^{1}}{\left|m-1-\beta_{20}^{1}\right|}$, is also identified. By assumption A12,

$$
\widetilde{\beta}(m) \equiv \frac{(m-1) \beta_{10}-\beta_{20}}{m-1-\beta_{20}^{1}}=\frac{\frac{(m-1) \beta_{10}-\beta_{20}}{\left|m-1-\beta_{20}^{1}\right|}}{\frac{(m-1) \beta_{10}^{1}-\beta_{20}^{1}}{\left|m-1-\beta_{20}^{1}\right|}},
$$

so that $\widetilde{\beta}(m)$ is identified as the ratio of two known terms. The rest of the proof of identification of $\left(\beta_{10}, \beta_{20}\right)$ follows the same line than the one of Theorem $1, \lambda_{0}$ being replaced by $-\beta_{20}^{1}$.

However, $\lambda_{0}$ cannot be identified. Indeed, let $\lambda_{0}^{\prime} \neq \lambda_{0}$ and define

$$
\varepsilon_{i}^{\prime}=\varepsilon_{i}+\bar{\varepsilon} \frac{m\left(\lambda_{0}-\lambda_{0}^{\prime}\right)}{\left(m-1+\lambda_{0}^{\prime}\right)\left(1-\lambda_{0}\right)} .
$$

Finally let

$$
\alpha^{\prime}=\frac{m \bar{x}\left(\beta_{10}+\beta_{20}\right)\left(\lambda_{0}-\lambda_{0}^{\prime}\right)+\alpha\left(m-1+\lambda_{0}\right)\left(1-\lambda_{0}^{\prime}\right)}{\left(m-1+\lambda_{0}^{\prime}\right)\left(1-\lambda_{0}\right)} .
$$

Then $\left(\lambda_{0}^{\prime}, \alpha^{\prime}, \varepsilon_{1}^{\prime}, \ldots, \varepsilon_{m}^{\prime}\right)$ are observationally equivalent to the initial model. Indeed, we can check that they lead to (4) as well. Moreover, conditioning on $\left(m, x_{1}, \ldots, x_{m}, \alpha^{\prime}\right)$ is equivalent to conditioning on $\left(m, x_{1}, \ldots, x_{m}, \alpha\right)$, and conditional exchangeability of $\left(\varepsilon_{1}, \ldots, \varepsilon_{m}\right)$ implies conditional exchangeability of the $\left(\varepsilon_{1}^{\prime}, \ldots, \varepsilon_{m}^{\prime}\right)$. Furthermore,

$$
F_{\varepsilon_{1}^{\prime}+\overline{\varepsilon^{\prime}} \frac{m}{m-1} \frac{\lambda_{0}^{\prime}}{1-\lambda_{0}^{\prime}} \mid m=m^{*}, x_{1}=x_{1}^{*}, \ldots, x_{m}=x_{m}^{*}, \alpha^{\prime}=\alpha^{\prime *}}=F_{\varepsilon_{1}+\bar{\varepsilon} \frac{m}{m-1} \frac{\lambda_{0}}{1-\lambda_{0}} \mid m=m^{*}, x_{1}=x_{1}^{*}, \ldots, x_{m}=x_{m}^{*}, \alpha=\alpha^{*}},
$$


where

$$
\alpha^{*}=\frac{\left(m-1+\lambda_{0}^{\prime}\right)\left(1-\lambda_{0}\right) \alpha^{*}-m \bar{x}\left(\beta_{10}+\beta_{20}\right)\left(\lambda_{0}-\lambda_{0}^{\prime}\right)}{\left(m-1+\lambda_{0}\right)\left(1-\lambda_{0}^{\prime}\right)} .
$$

Thus the second part of assumption A10 also holds with $\left(\lambda_{0}^{\prime}, \alpha^{\prime}, \varepsilon_{1}^{\prime}, \ldots, \varepsilon_{m}^{\prime}\right)$. This shows that $\lambda_{0}$ is not identified.

\section{Theorem 5}

Let $\theta_{0}=\frac{\lambda_{0}}{1-\lambda_{0}}$ and

$$
v_{i}=\left[\bar{x} \frac{m}{m-1}\left[\beta_{20}+\theta_{0}\left(\beta_{10}+\beta_{20}\right)\right]+\alpha\left(1+\frac{m}{m-1} \theta_{0}\right)\right]+\bar{\varepsilon} \frac{m}{m-1} \theta_{0}+\varepsilon_{i} .
$$

Note that $F_{v_{1}, \ldots, v_{m} \mid x_{1}, \ldots, x_{m}, m}=F_{v_{1}, \ldots, v_{m} \mid \bar{x}, m}$. Indeed

$$
\begin{aligned}
F_{v_{1}, \ldots, v_{m} \mid x_{1}, \ldots, x_{m}, m}\left(v_{1}^{*}, \ldots, v_{m}^{*} \mid x_{1}^{*}, \ldots, x_{m}^{*}, m^{*}\right) \\
\quad=\int F_{v_{1}, \ldots, v_{m} \mid x_{1}, \ldots, x_{m}, m, \alpha}\left(v_{1}^{*}, \ldots, v_{m}^{*} \mid x_{1}^{*}, \ldots, x_{m}^{*}, m^{*}, \alpha^{*}\right) d F_{\alpha \mid x_{1}, \ldots, x_{m}, m}\left(\alpha^{*} \mid x_{1}^{*}, \ldots, x_{m}^{*}, m^{*}\right) \\
\quad=\int F_{v_{1}, \ldots, v_{m} \mid \bar{x}, \alpha, m}\left(v_{1}^{*}, \ldots, v_{m}^{*} \mid \overline{x^{*}}, \alpha^{*}, m^{*}\right) d F_{\alpha \mid \bar{x}, m}\left(\alpha^{*} \mid \overline{x^{*}}, m\right) \\
\quad=F_{v_{1}, \ldots, v_{m} \mid \bar{x}, m}\left(v_{1}^{*}, \ldots, v_{m}^{*} \mid \overline{x^{*}}, m^{*}\right),
\end{aligned}
$$

where the third line stems from assumption A13 and the fact that, given $x_{1}, \ldots, x_{m}, m, \alpha$, $\left(v_{1}, \ldots, v_{m}\right)$ is a deterministic function of $\left(\varepsilon_{1}, \ldots, \varepsilon_{m}\right)$. Now

$$
\begin{aligned}
& \operatorname{Pr}\left(y_{1}=0, y_{2}=0 \mid x_{1}=x_{1}^{*}, x_{2}=x_{2}^{*}, \bar{x}=x, m=m^{*}\right) \\
& =\operatorname{Pr}\left\{v_{1} \leq-x_{1}^{*}\left(\beta_{10}-\frac{\beta_{20}}{m-1}\right), v_{2} \leq-x_{2}^{*}\left(\beta_{10}-\frac{\beta_{20}}{m-1}\right) \mid x_{1}=x_{1}^{*}, x_{2}=x_{2}^{*}, \bar{x}=x, m=m^{*}\right\} \\
& =F_{v_{1}, v_{2} \mid \bar{x}, m}\left(-x_{1}^{*}\left(\beta_{10}-\frac{\beta_{20}}{m^{*}-1}\right),-x_{2}^{*}\left(\beta_{10}-\frac{\beta_{20}}{m^{*}-1}\right) \mid x, m^{*}\right) .
\end{aligned}
$$

Because, by theorem $4,\left(\beta_{10}, \beta_{20}\right)$ is identified, $x_{1}^{*}\left(\beta_{10}-\frac{\beta_{20}}{m^{*}-1}\right)$ and $x_{2}^{*}\left(\beta_{10}-\frac{\beta_{20}}{m^{*}-1}\right)$ are known. Moreover, $\bar{x}$ is observed so that the first term is identified on the whole support of $\left(x_{1}, x_{2}\right)$. Thus, by assumption A15, making $\left(x_{1}, x_{2}\right)$ vary allows us to recover the whole conditional distribution of $\left(v_{1}, v_{2}\right)$ given $\bar{x}$ and $m$. Thus, using assumption A14,

$$
\operatorname{Cov}\left(v_{1}, v_{1}-v_{2} \mid \bar{x}, m\right)=\operatorname{Cov}\left(\bar{\varepsilon} \frac{m}{m-1} \theta_{0}+\varepsilon_{1}, \varepsilon_{1}-\varepsilon_{2} \mid \bar{x}, m\right)=\operatorname{Var}\left(\varepsilon_{1} \mid \bar{x}\right),
$$

so that the right term is identified. By the way, let us note that assumption A14 can be tested thanks to the data, since it implies that $\operatorname{Cov}\left(v_{1}, v_{1}-v_{2} \mid \bar{x}, m\right)$ does not depend on $m$. 
Moreover, a little algebra shows that

$$
\begin{aligned}
(m-1)^{2} \operatorname{Cov}\left(v_{1}, v_{2} \mid \bar{x}, m\right)= & m^{2}\left[\left(1+\theta_{0}\right)^{2} \operatorname{Var}(\alpha \mid \bar{x})\right]+m\left[-2\left(1+\theta_{0}\right) \operatorname{Var}(\alpha \mid \bar{x})\right. \\
& \left.+\theta_{0}\left(2+\theta_{0}\right) \operatorname{Var}\left(\varepsilon_{1} \mid \bar{x}\right)\right]+\left[\operatorname{Var}(\alpha \mid \bar{x})-2 \theta_{0} \operatorname{Var}\left(\varepsilon_{1} \mid \bar{x}\right)\right] .
\end{aligned}
$$

This is a regression of the (known) left term on $\left(m^{2}, m, 1\right)$. Because $m$ takes at least three values, the coefficients $(a, b, c)$ of this regression can be recovered. ${ }^{15}$ We will show that the knowledge of these coefficients implies that $\theta_{0}$ is identified. The conclusion will follow because $\theta_{0}$ is one-to-one with $\lambda_{0}$.

First, let $\phi_{0}=1+\theta_{0}$ and $\rho_{0}=\frac{\operatorname{Var}(\alpha \mid \bar{x})}{\operatorname{Var}(\varepsilon \mid \bar{x})}$. Let also $a^{\prime}=a / \operatorname{Var}(\varepsilon \mid \bar{x}), b^{\prime}=b / \operatorname{Var}(\varepsilon \mid \bar{x})+1$ and $c^{\prime}=c / \operatorname{Var}(\varepsilon \mid \bar{x})-2$. Then $a^{\prime}, b^{\prime}$ and $c^{\prime}$ are identified, and

$$
\left\{\begin{aligned}
\phi_{0}^{2} \rho_{0} & =a^{\prime} \\
-2 \phi_{0} \rho_{0}+\phi_{0}^{2} & =b^{\prime} \\
\rho_{0}-2 \phi_{0} & =c^{\prime} .
\end{aligned}\right.
$$

Replacing $\rho_{0}$ by $c^{\prime}+2 \phi_{0}$ in the first and second equation leads to

$$
\left\{\begin{aligned}
\phi_{0}^{3}+c^{\prime} / 2 \phi_{0}^{2}-a^{\prime} / 2 & =0 \\
\phi_{0}^{2}+2 c^{\prime} / 3 \phi_{0}+b^{\prime} / 3 & =0 \\
\rho_{0}-2 \phi_{0} & =c^{\prime} .
\end{aligned}\right.
$$

This system admits at most two solutions in $(\rho, \phi)$. Suppose that there are two different solutions, and let $\left(\rho_{1}, \phi_{1}\right)$ denote the second one. Then we can write the polynomial of the first equation as a product in which one factor is the polynomial of the second equation. Hence, there exists $x$ such as, for all $\phi \in \mathbb{R}$,

$$
\phi^{3}+c^{\prime} / 2 \phi^{2}-a^{\prime} / 2=\left(\phi^{2}+2 c^{\prime} / 3 \phi^{2}+b^{\prime} / 3\right)(\phi+x) .
$$

Thus

$$
\left\{\begin{aligned}
x & =-c^{\prime} / 6 \\
2 c^{\prime} x & =-b^{\prime} \\
2 b^{\prime} x & =-3 a^{\prime} .
\end{aligned}\right.
$$

Hence $c^{\prime 2}=3 b^{\prime}$. Replacing $b^{\prime}$ and $c^{\prime}$ by their expression gives

$$
3\left(-2 \phi \rho+\phi^{2}\right)=(\rho-2 \phi)^{2}
$$

which must hold for $\left(\rho_{0}, \phi_{0}\right)$ and $\left(\rho_{1}, \phi_{1}\right)$. But this statement is equivalent to $\phi+\rho=0$. Replacing $\rho$ by $-\phi$ in $c^{\prime}$ gives $\phi_{0}=\phi_{1}=-c^{\prime} / 3$ and thus also $\rho_{0}=\rho_{1}$. This contradicts $\left(\rho_{0}, \phi_{0}\right) \neq\left(\rho_{1}, \phi_{1}\right)$. Thus $\phi_{0}$ is identified by 8 and the conclusion follows.

\footnotetext{
${ }^{15}$ These coefficients depend on $\bar{x}$ but for the sake of simplicity, we let this dependency implicit from now on.
} 


\section{Proposition 1}

Let $k_{r i}=x_{r i} \beta_{10}+\left(\frac{1}{m_{r}-1} \sum_{j=1, j \neq i}^{m_{r}} x_{r j}\right) \beta_{20}+\alpha_{r}+\varepsilon_{r i}, Y_{r}^{t}=\left(y_{r 1}^{t}, \ldots, y_{r m_{r}}^{t}\right)^{\prime}$ and $K_{r}=$ $\left(k_{r 1}, \ldots, k_{r m_{r}}\right)^{\prime}$. Equation (2) can be rewritten in matrix terms:

$$
\frac{d Y_{r}^{t}}{d t}=\left[\left(\mu-\frac{\lambda_{0}}{m_{r}-1}\right) I_{m_{r}}+\frac{\lambda_{0}}{m_{r}-1} J_{m_{r}} d\right] Y_{r}^{t}+K_{r},
$$

where $J_{m_{r}}$ denotes the matrix of ones of size $m_{r}$. The eigenvalues of $A_{r}=\left(\mu-\frac{\lambda_{0}}{m_{r}-1}\right) I_{m_{r}}+$ $\frac{\lambda_{0}}{m_{r}-1} J_{m_{r}}$ are $\mu-\frac{\lambda_{0}}{m_{r}-1}$ and $\mu+\lambda_{0}$. Thus the system is stable when $\max \left(\mu-\frac{\lambda_{0}}{m_{r}-1}, \mu+\lambda_{0}\right) \leq 0$. Because $\max \left(\mu-\frac{\lambda_{0}}{m_{r}-1}, \mu+\lambda_{0}\right) \geq \mu$, this implies that $\mu \leq 0$. Moreover, when $\lambda_{0} \geq 0$, the condition is equivalent to $\lambda_{0} \leq-\mu$ and when $\lambda_{0}<0$, this amounts to $\lambda_{0} \geq \mu\left(m_{r}-1\right)$. Thus, the system is stable for all $r=1 \ldots R$ if and only if

$$
\mu \leq 0 \text { and } \lambda_{0} \in\left[\mu\left\{\min _{r}\left(m_{r}\right)-1\right\},-\mu\right] .
$$

If these conditions are fulfilled, then, $\lim _{t \rightarrow+\infty} A_{r} Y_{r}^{t}=-K_{r}$. Equivalently, if $y_{r i}$ denotes the $i$-th component of the stationary state of $Y_{r}^{t}$,

$$
-\mu y_{r i}=\left(\frac{1}{m_{r}-1} \sum_{j=1, j \neq i}^{m_{r}} y_{r j}\right) \lambda_{0}+x_{r i} \beta_{10}+\left(\frac{1}{m_{r}-1} \sum_{j=1, j \neq i}^{m_{r}} x_{r j}\right) \beta_{20}+\alpha_{r}+\varepsilon_{r i} .
$$

Thus, when $\mu=-1$, we retrieve equation (1).

\section{References}

Ammermueller A. and J. S. Pischke, 2006, Peer Effects in European Primary Schools: Evidence from PIRLS. IZA Discussion Paper No. 2077.

Angrist J. D. and K. Lang, 2004, Does School Integration Generate Peer Effects? Evidence from Boston's Metco Program. The American Economic Review 94, 1613-1634.

Bayer, P. and C. Timmins, , 2002, Identifying Social Interactions in Endogenous Sorting Models. Working Paper, Department of Economics, Yale University.

Brock W. A. and S. M. Durlauf, 2001, Discrete Choice with Social Interactions. Review of Economic Studies 68, 235-260.

Brock W. A. and S. M. Durlauf, 2004, Identification of Binary Choice Models with Social Interactions. Working Paper, Wisconsin Madison - Social Systems. 
Cooley J., 2006, Desegregation and the Achievement Gap: Do Diverse Peers Help? Working Paper, Duke University.

Duflo E. and E. Saez, 2002, The Role of Information and Social Interactions in Retirement Plan Decisions: Evidence from a Randomized Experiment. NBER Working Paper No 8885 .

Graham B. S., 2005, Identifying Social Interactions through Excess Variance Contrasts. Working Paper, Harvard University.

Graham B. S. and J. Hahn, 2005, Identification and Estimation of the Linear-in-Means Model of Social Interactions. Economics Letters 88, 1-6.

Hoxby C., 2000, Peer Effects in the Classroom: Learning from Gender and Race Variation. NBER Working Paper No 7867.

Krauth B. V., 2006, Simulation-based estimation of peer effects. Journal of Econometrics $133,243-271$.

Lee L. F., 2006, Identification and Estimation of Econometric Models with Group Interactions, Contextual Factors and Fixed Effects. Journal of Econometrics, forthcoming.

Lewbel A., 1997, Constructing Instruments for Regressions With Measurement Error When no Additional Data are Available, with an application to Patents and R \& D. Econometrica 65, 1201-1213.

Lewbel A., 2000, Semiparametric Qualitative Response Model Estimation With Unknown Heteroscedasticity and Instrumental Variables. Journal of Econometrics 97, 145-177.

Manski C., 1987, Semiparametric Analysis of Random Effects Linear Models from Binary Panel Data. Econometrica 55, 357-362.

Manski C., 1993, Identification of Endogenous Social Effects: the Reflection Problem. Review of Economic Studies 60, 531-542.

Manski C., 2000, Economic Analysis of Social Interactions. Journal of Economic Perspectives $14,115-136$. 
Rees D. I., J. S. Zax and J. Herries, 2003, Interdependence in worker productivity. Journal of Applied Econometrics 18, 585-604.

Schennach S., 2004, Estimation of Nonlinear Models with Measurement Error. Econometrica $72,33-75$.

Tamer E., 2003, Incomplete Simultaneous Discrete Response Models with Multiple Equilibria. Review of Economic Studies 70, 147-165. 